\title{
LINE-DRAWING ENHANCED INTERACTIVE MURAL RESTORATION FOR DUNHUANG MOGAO GROTTOES
}

\author{
X. Y. Fu ${ }^{\text {a }}$ Y. Han ${ }^{\text {a }}$ Z. J. Sun ${ }^{\text {b }, ~ X . ~ J . ~ M a ~}{ }^{\text {c, } * \text {, Y. Q. Xu }}{ }^{\text {a, } *}$ \\ ${ }^{a}$ Academy of Arts \& Design, Tsinghua University, Beijing, China - (fuxy15, hanyi14) @mails.tsinghua.edu.cn, \\ yqxu@tsinghua.edu.cn \\ b Dunhuang Research Academy, Dunhuang, Gansu, China - sunzj@dha.ac.cn \\ ${ }^{c}$ Department of Computer Science and Engineering, the Hong Kong University of Science and Technology, Clear Water Bay,
} Kowloon, Hong Kong-mxj@cse.ust.hk

\section{Commission II}

KEY WORDS: Dunhuang Mogao Grottoes, Mural, Restoration, Line Drawing, Interaction

\begin{abstract}
:
Dunhuang Mogao Grottoes in western China is one of the most famous World Cultural Heritage Sites, known for its glorious Chinese Buddhist art spanning a period of 1,000 years. However, it has been suffering from damage and degradation caused by man-made and natural factors. In this article, we present a novel line-drawing enhanced interactive system for digital restoration of damaged murals in Mogao Grottoes. Our system consists of four components, namely data pre-processing, damaged area selection, line-drawing segmentation, and mural restoration. Each component is a hybrid of efficient algorithms and user interactions. We introduce the infrastructure and process of using our system, from data capture and collection, database establishment, to interactive restoration. We conduct a user study with 15 participants who have varied experiences with and skills on repairing murals and editing images. Results and feedback suggest that our system can achieve satisfactory restoration results without overburdening the users. It can benefit both experts trained in restoration and amateurs interested in cultural heritage conservation.
\end{abstract}

\section{INTRODUCTION}

Murals are one of the most valuable heritages for studying ancient history, as it preserves a faithful pictorial record of the lives and beliefs at the time. Existing murals have given archaeologists important insights into the economics, politics, religions, and cultures in the past. Dated back to 1,600 years ago, the Dunhuang Mogao Grottoes is a shrine of splendid Chinese Buddhist artwork spanning a period of 1,000 years with more than ten genres of murals and statues (Whitfield et al., 2015). Figure 1 shows the exquisite mural of Amitabha Sutra in Mogao Grottoes, south wall, Cave 220.

Unfortunately, most of the existing murals are suffering from degradations due to historical or natural reasons, such as chemical reaction (Sayre, 1973), water, wind, salt erosion, vegetation, and fungal influence (Milanesi et al., 2006). In such cases of Dunhuang Mogao Grottoes, which is located in a desertification area (Wang et al., 2000), damages found on its murals include flaking (Ma and Wang, 2014), disruption (Fan et al., 2009), blister (Chen and Yu, 2008), etc. Researchers have been using suitable material and techniques to physically repair some of the flaking, disruption, and blister on Dunhuang murals (Fan et al., 2008) (Fan et al., 2009). However, such physical restoration projects aim at controlling and relieving further deterioration rather than restoring color and content of murals. This is a task that requires exquisite skills and accurate operations to avoid manual damage to murals.

Digital restoration represents a repeatable and efficient approach that requires minimal physical interactions with murals. Dunhuang murals often illustrate a story with complex, nonlinear composition of many characters, architectures, musical

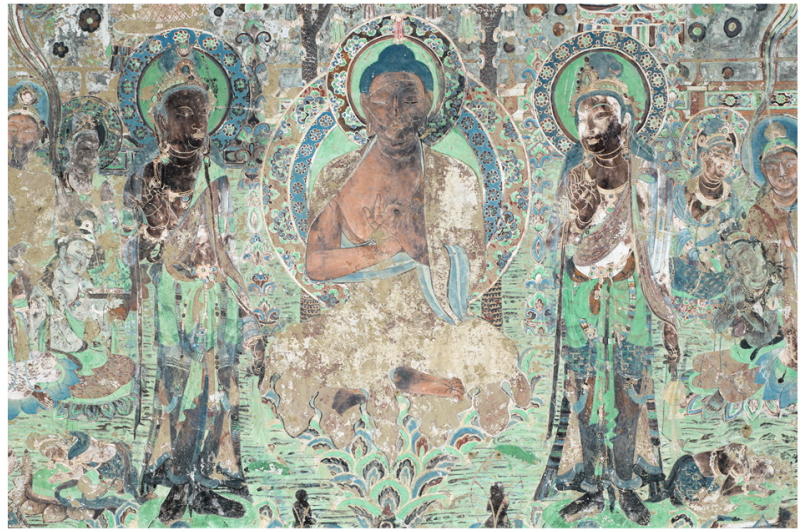

Figure 1. Mogao Cave 220, south wall, Amitabha Sutra.

instruments, animals, and natural sceneries. Complex content and extensive size of damaged areas make it difficult for many solutions (e.g., (Blazek et al., 2009), (Mastio et al., 2007), (Pappas and Pitas, 2000)), using automatic image processing algorithms like edge extraction, to detect damage and segment regions. In such a cases, manual supervision and correction is then necessary, generating intensive and time consuming labour.

Fortunately, there exist line drawings of some of the Dunhuang murals on paper (Fraser, 2000) (Cahill et al., 2001). These illustrations can serve as valuable references for digital restoration. Built upon these facts, we introduce an infrastructure for digitally repairing damaged murals in Dunhuang Mogao Grottoes, providing data collection, database establishment, and interactive restoration that leverages existing line drawings. Our

\footnotetext{
* Corresponding author
} 
restoration results can serve as references for physical restoration and art reproduction of murals. Experimental results show that our system can achieve good interactive experience, both for experts and amateurs.

In the rest of the paper, we first review related work on mural restoration in Section 2. In section 3, we provide an overview and details of our line-drawing enhanced interactive system for Dunhuang mural restoration. Section 4 shows the validation and evaluation results of our system with both experts and amateur users. In section 5, we discuss the limitation and practical applications of this work, followed by conclusion and future work in Section 6.

\section{RELATED WORK}

Restoration of murals aims at repairing the deteriorated parts while maintaining the original condition of murals to the best extent. The state-of-art techniques can be divided into physical restoration and digital restoration.

\subsection{Physical restoration}

Physical restoration concerns controlling and relieving deterioration of a mural. Common techniques include cleaning (Carretti et al., 2005), grouting (Baglioni et al., 1997), reinforcement (Li et al., 2013), desalination (Chen et al., 2009), etc.

As for Dunhuang Mogao Grottoes, conservators follow the principle of keeping the original state of murals when applying restoration. The physical restoration of Dunhuang Mogao Grottoes started in 1951 (Li, 2004). Over the years, conservators have developed customized materials, tools, and techniques to handle the different types of damages, such as detachment, disruption and flaking (Fan et al., 2008) (Fan et al., 2009). Physical restoration requires the conservators to be highly concentrated and skillful to prevent secondary damage, because direct touch is extremely dangerous especially for murals in very bad conditions.

\subsection{Digital Restoration}

Digital restoration can recover the color and content of murals with minimal physical interactions. Technical problems of digital restoration consist of two steps: how to detect or select damaged region, and how to fill the region up with proper pixels. Underlying algorithms include defect detection, image segmentation, image inpainting and texture synthesis.

\subsection{Digital Restoration}

Digital restoration can recover the color and content of murals with minimal physical interactions. Technical problems of digital restoration consist of two steps: how to detect or select damaged region, and how to fill the region up with proper pixels. Underlying algorithms include defect detection, image segmentation, image inpainting and texture synthesis.

Defects detection aims to separate regions to be fixed from intact areas. Traditionally, it is done manually, which is subjective and enervating (Padalkar and Joshi, 2015) (Turakhia et al., 2012). Researchers have tried to automate this process, at least partially. They exploited color information to locate defects on images automatically (Chang et al., 2005) (Amano, 2006) or semiautomatically (Giakoumis et al., 2006). For example, Chang's mechanism makes use of signal filters and structural information.
Amano proposed a correlation-based approach to extract the image description rule from local regions. These methods can identify defective areas that are salient and related, but often fail when distinctions between defects and the image are not obvious.

Large sized images need to be perceptually segmented before being processed. There are plenty of advanced techniques to solve this problem which can be divided into edge-based, regionbased and hybrid segmentation algorithms. Edge-based segmentation rely on the discontinuity and abrupt changes of boundaries in gray-scale level (Palmer et al., 1996), and need additional algorithms like gap filling and smoothing to close the extracted curves, which would be rather time consuming. Region-based segmentation utilize the similar properties of pixels in gray or color value to produce meaningful regions. Representative technology is seeded region growing algorithm (Adams and Bischof, 1994). It groups the pixels according to the criteria and given seeds and gains better results than edge-based methods, but the initial seed should be selected accurately. Hybrid segmentation technologies combine multiple methods to gain more accurate results. (Fan et al., 2001) proposes a hybrid method by integrating the results of color-edge extraction and seeded region growing. (Achanta et al., 2012) introduces the simple linear iterative clustering (SLIC) algorithm, which adapts a k-means clustering approach to efficiently generate superpixels. As for Dunhuang murals, extensive damage areas have destroyed the integrity of image both in edge and color, traditional methods often fail to make satisfactory segmentation results.

Image inpainting algorithms can be applied to the selected regions of interest to reconstruct their structures and fill up the pixels with colors closed to the original. Existing inpainting algorithms for removing unwanted elements from an image, such as text, logos, and signature, etc., can be based on texture synthesis (Efros and Leung, 1999) (Harrison, 2001), Partial Differential Equation (PDE) (Bertalmio et al., 2003) (Chan and Shen, 2002), given exemplar (Criminisi et al., 2004) (Drori et al., 2003), or a combination of them (Starck et al., 2005). However, these approaches may not be suitable for mural restoration. Texture synthesis, in fact, is more suitable for images with simple repeated patterns such as brick walls, feathers, and textiles. Partial-Differential-Equation-based methods may introduce blurry artifacts and thus are less commonly used these days. Exemplar-based approaches can remove large objects from a target image and fill the hole with textures and structure from specified exemplars. In this paper, we adapt the exemplar-based method and tailor it to meet the characteristics of Dunhuang murals.

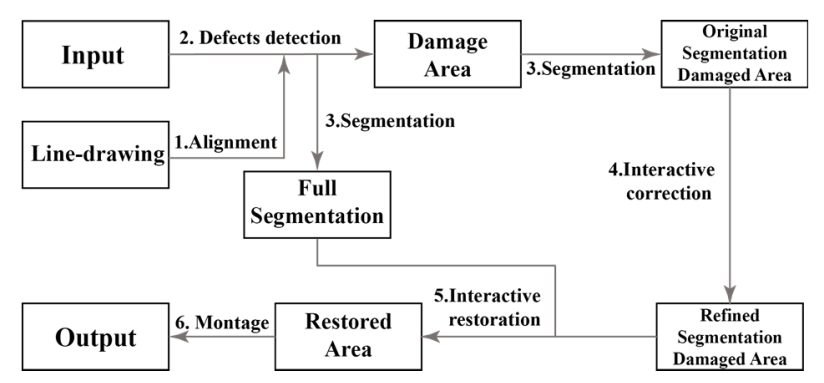

Figure 2. Interactive system flowchart.

\section{INTERACTIVE MURAL RESTORATION}

In this article, we propose a digital mural restoration infrastructure that supports data capturing and processing, database establishment, and interactive reconstruction for 


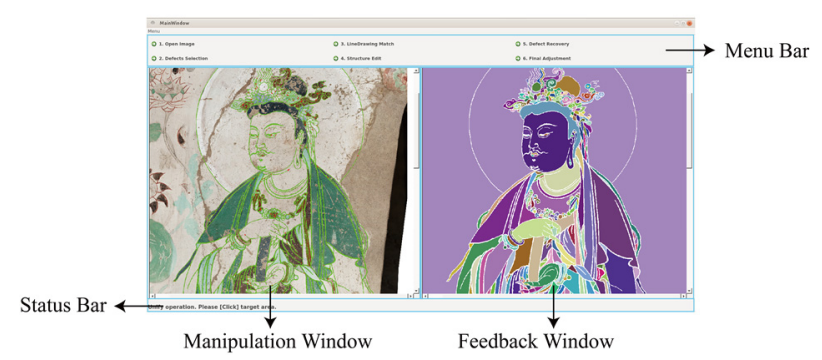

Figure 3. System interface. The system consists of a menu bar, two vertical split windows and a status bar.

Dunhuang murals. We design the system in a way that it is easy to learn and master by both experts and amateurs.

\subsection{Overview of system workflow}

The interactive restoration system consists of data pre-processing, damaged area selection, line-drawing segmentation, and mural restoration. As stressed above, Dunhuang murals are complex in content and have large amounts of damaged areas, which makes it very difficult for traditional image processing approaches to solve the restoration problem automatically. Thus, we try to involve aids of users by combining interactions with classical image processing algorithms in each steps and results show that our approach gains satisfactory restoration effects, which can't be obtained through traditional ways.

As illustrated in Figure 2, our system works like this: once the mural image and its associated line drawing are loaded from the database, we align them together semi-automatically by moving, rescaling, and rotating the contour illustration overlaid on top of the mural image. Then users iteratively pick out the deteriorated regions with the assistance of our system's damaged area selection algorithm. The next step is to separate the different visual elements in the mural. The initial segmentation result is automatically generated using the structure information of the line drawing. It may have flaws, depending on the quality of the drawing. Users can interactively refine the segmentation results. After that, users can specify a reference color and a treatment method to algorithmically repaint the target area.

Figure 3 shows an overview of our system interface. We keep a simple and clean style to minimize visual distractions. The interface consists of a menu bar, two vertical split screens, and a status bar. The menu bar lists the six steps listed in Figure 2 in a sequential order to guide users through the process. The two vertical split screens with synchronous scrolling and scaling enable users to compare the input (left screen) and the output (right screen) after each manipulation. All the user interactions with proper visual feedback are conducted on the left screen, such as clicking and dragging. Users can check the intermediate and final states of the restored image on the right screen. The status bar informs user the progress of the algorithms running in the backend of the system.

\subsection{Data Collection and Database Establishment}

Our system contains a database of mural images and a database of line drawings.

Digital Mural Database. We have captured three types of digital images of Mogao Cave 156/159/172/254/320, details are shown in Table 1. We build a soft, uniform lighting environment for the photo taking process to maintain the primitiveness of content on the images. We use X-Rite Color Checker to calibrate the digital color and panoramic photography for image stitching.

\begin{tabular}{|c|c|c|c|c|c|}
\hline Type & Amount & Size & $\begin{array}{c}\text { Resol } \\
\text { ution }\end{array}$ & Camera & Lens \\
\hline $\begin{array}{c}\text { Multiple } \\
\text { photos } \\
\text { for } \\
\text { stitching }\end{array}$ & 542 & $\begin{array}{c}5760 \\
*\end{array}$ & $\begin{array}{c}72 \\
\text { dpi }\end{array}$ & $\begin{array}{c}\text { Canon } \\
\text { EOS 5D } \\
\text { Mark III }\end{array}$ & $\begin{array}{c}\text { Canon } \\
24-70\end{array}$ \\
\hline $\begin{array}{c}\text { Detailed } \\
\text { photos of } \\
\text { selected } \\
\text { parts }\end{array}$ & 134 & $\begin{array}{c}* 184 \\
*\end{array}$ & $\begin{array}{c}72 \\
\text { dpi }\end{array}$ & $\begin{array}{c}\text { EOS 5D } \\
\text { Mark III }\end{array}$ & $\begin{array}{c}\text { Canon } \\
\text { and } \\
70-200\end{array}$ \\
\hline $\begin{array}{c}\text { Full } \\
\text { frame } \\
\text { photos }\end{array}$ & 45 & $\begin{array}{c}8272 \\
*\end{array}$ & $\begin{array}{c}300 \\
\text { dpi }\end{array}$ & $\begin{array}{c}\text { Hasselbl } \\
\text { ad H5D- } \\
40\end{array}$ & $\begin{array}{c}\text { HC } \\
2.8 / 80 \\
\mathrm{~mm}\end{array}$ \\
\hline
\end{tabular}

Table 1. Information of digital mural database.

Line-drawing Database. We digitalize a totally 195 pieces of line drawings from different sources, such as books and unpublished artwork by Dunhuang artists. These drawings are usually large in size and thus cannot be scanned all at once. Instead, we applied the photo stitching technique to combine images of different segments into one piece. The line drawings are mostly produced on Chinese rice paper which is very sensitive to wrinkles, as shown in Figure 4 (a). The digital copy line drawing may contain shades of various intensities as a result of the wrinkles and the lighting condition. Besides, the lines are drawn in graded ink and thus are not uniform in color. Some of the lines are even lighter than the shades, making it difficult to extract the meaningful contours using simple algorithms. We observe that the shades tend to occupy a large area with slow variation in gray-scale values, while lines are thinner structures and its change of gray value in certain direction is rather rapid. Therefore, we apply a median filter to obtain the parts of shadows and wrinkles. Then we subtract these areas from the original copy and convert the remaining to a binary black-and-white image. The result of line drawing digitalization is shown in Figure 4(b).

\subsection{Pre-processing}

In this paper, we use the Bodhisattva of Cave 320 (Figure 4(c)) to demonstrate the process and performance of our proposed system. Cave 320 was painted in Tang Dynasty, and is representative in terms of content and style. Besides, the Bodhisattva suffers from various degradations to different extent, but the whole figure remains relatively complete and most of the elements are identifiable. We selected the high quality line drawing of Bodhisattva in Cave 320 painted by artists from Dunhuang Research Academy in China (Figure 4(a)).

Before being presented to users, each pair of line drawing and mural image in our system needs to be aligned together (Figure 4(d)). Since the experimental binary image of line drawing almost align with the fresco, we apply the simple affine transformation method to optimize the alignment. That is, we choose three couples of key points on the fresco image and the line-drawing image, then we calculate the parameters of affine transformation matrix according to the chosen key points, finally we apply the affine transformation to the line-drawing image so that it can scale, rotate and translate to fit the fresco image. Note that if the line-drawing image is very different from the mural image, the alignment turns out to be difficult and not suitable to be done through simple algorithms. More advanced algorithms 


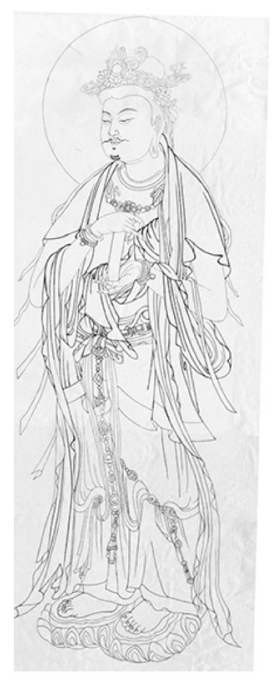

(a)

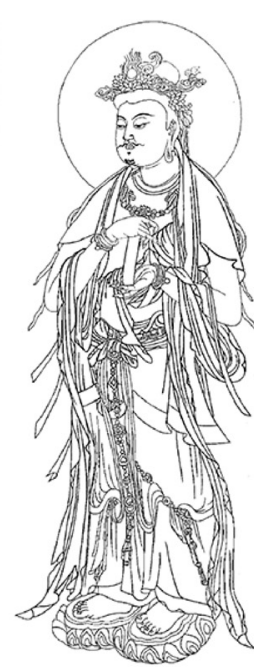

(b)

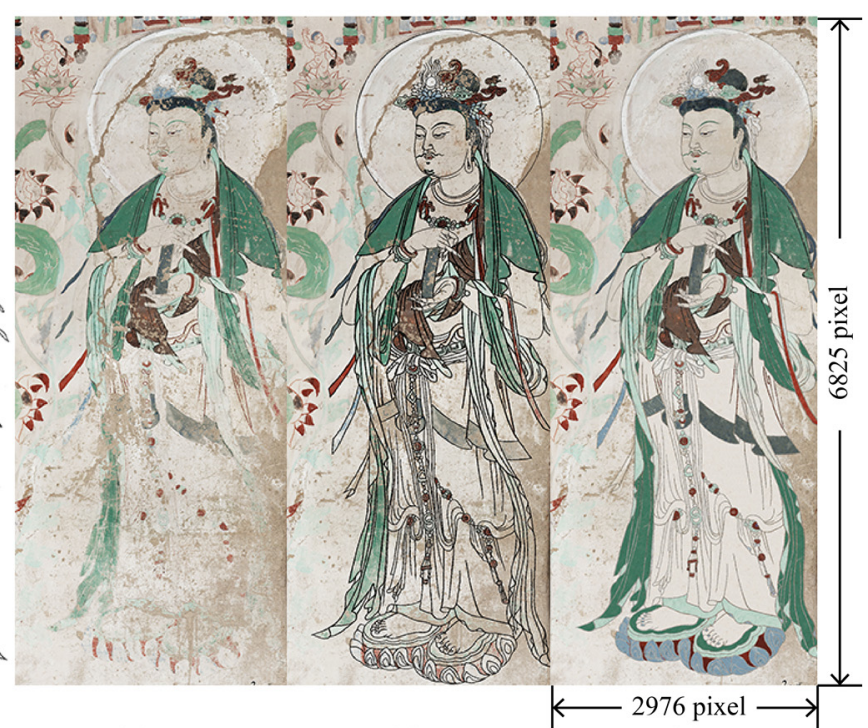

(d)

(e)

Figure 4. (a) Original line drawing on Chinese rice paper; (b) digitalized line drawing after pre-processing; (c) photo of original mural; (d) alignment of line drawing and mural image; (e) restoration result.

can be found in (Szeliski, 2006) (Russell et al., 2011) to deal with complex cases.

\subsection{Damaged Area Selection}

With the help of a RGB color-based algorithm running in the backend, users can interactively pick out the damaged areas. With a few clicks in a damaged area, all pixels within a certain color range of the selected pixels will be automatically highlighted consequently. The color range is determined by a threshold, user can manually adjust its value or simply use the default number. Users can save the result and perform these actions iteratively until they are satisfied with the aggregated outcome.

RGB color-based defects selecting strategy. Setting a threshold $t$ and click at one pixel $p_{c}$ each time. The color of $p$ is denoted as $r_{c} g_{c} b_{c}$, and the color range $C(t)$ is defined as equation (1). The size of the set is calculated as equation (2). The system then traverses all pixels in the image and selects $\forall p, r_{p} g_{p} b_{p} \in C(t)$.

$$
\begin{aligned}
\mathrm{C}(\mathrm{t})=\{\mathrm{rgb} \mid \mathrm{r} & \in\left[\mathrm{r}_{\mathrm{c}}-\mathrm{t}, \mathrm{r}_{\mathrm{c}}+\mathrm{t}\right], \\
\mathrm{g} & \in\left[\mathrm{g}_{\mathrm{c}} \mathrm{t}, \mathrm{g}_{\mathrm{c}}+\mathrm{t}\right], \\
\mathrm{b} & \left.\in\left[\mathrm{b}_{\mathrm{c}}-\mathrm{t}, \mathrm{b}_{\mathrm{c}}+\mathrm{t}\right]\right\} \\
|\mathrm{C}(\mathrm{t})| & =(2 \mathrm{t}+1)^{3}
\end{aligned}
$$

The choice of color range has a direct impact on the effectiveness of the selection algorithm. We experiment with different parameter configurations to infer a proper $t$ as the default setting. We use the precision and recall of selection results to quantize the performance. Note that, we want to reduce the false positive rate, so that the well-preserved areas would not get treated by mistake. As shown in Figure 5 (b) and (c), we achieve the highest precision when $t=5$, and the highest recall when $t=7$. But we can see that when $t=7$, it introduces more false positive points in the face area compared to the hand-labeled ground truth (Figure 5 (a), in red and blue frames). Hence, we set the default threshold for color range inference to $t=5$. We also allow users to manually adjust the value before each click according to their needs. Users can increase the threshold to shorten the processing time or lower it to more fine-grained selection. In the future, we will further explore the potential to automatically adjust the threshold based on the number of clicks, so that users who have little knowledge about setting the threshold in person can also benefit from dynamic configuration.

\subsection{Line-drawing Segmentation}

Region segmentation aims to separate visual components with different colors and textures. There are plenty of advanced techniques to solve this problem as described in section 2. Our expected outputs are segmentations of entire perceptive meaningful regions with clear and occlusive boundaries. Traditional algorithms, like level sets (Tsai et al., 2003) (Paragios and Deriche, 2000) algorithm and k-means (Ray and Turi, 1999) approach can't meet our requirements of segmenting visual components because of the noise information of defects.

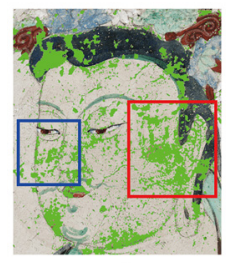

$t=7$

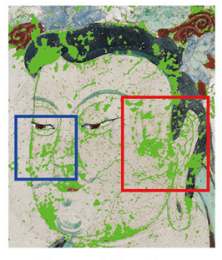

Ground Truth

(a)

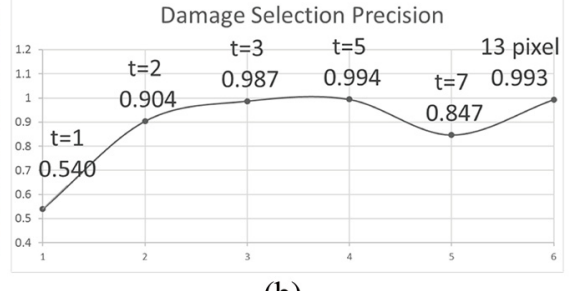

(b)

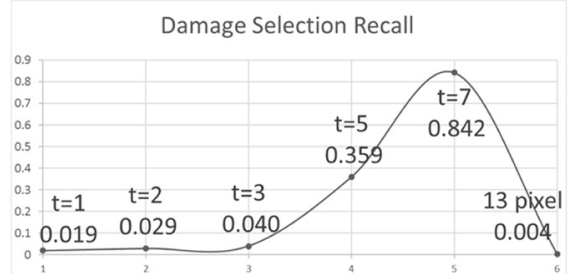

(c)
Figure 5. Experimental results of precision and recall. Figure (a) shows partial damage selection result when $t=7$ (left) and ground truth (right), defects are shown in green color. For five clicks results, Figure (b) shows the curve of precision and Figure (c) shows the curve of recall. 
Inspired by the work of (Blazek et al., 2009), who utilize ultraviolet spectrum technology to detect defects and laid the results on the mural image to segment it, we started to segment the line drawing by applying connected-component analysis and using the results to segment the mural image. There are two classical algorithms for connected-component analysis, i.e., seed-filling (Vincent and Soille, 1991) and two-pass (Rosenfeld and Pfaltz, 1966). Seed-filling is a recursive labeling algorithm. It starts with a 'seed' pixel in the target region and recursively expands the label to all the non-border neighbors. This process is rather slow, and thus we choose to use the two-pass method in this work.

Two-pass is a row-by-row labeling algorithm. It scans the input binary image twice, and keeps a record of different sets of equivalence classes throughout the process. The first pass is to label all the non-border pixels. For each pixel, if none of its neighbors are marked by a number, it gets a new numerical label. Otherwise, it takes the minimum label of its neighbors. Additionally, the algorithm puts the pixel and its neighbors into the same set of equivalence classes. The labels received after the first pass are only temporary, since there may be more than one labels in one connected region. The second pass is to find the minimum label in each set of equivalence classes and use it to replace all the temporary labels of the members in the set. The original result of region segmentation is shown in Figure 6(a).

However, over segmentation may occur due to the complex structure of the mural, while gaps on the contours extracted from the line drawing may mistakenly connect separate regions. Therefore, our system provides two operations, region splitting and region merging, for users to interactively modify the autosegmentation result.

Region Splitting. This function is for users to correct misconnected components or obtain more detailed sub-regions for finer operations. To perform this action, user need to first point out the problematic region by a left click. The selected region and the line(s) with gap(s) inside it get highlighted. Then, users can close an unwanted gap by drawing a line that starts and ends on the existing contour. Later, users can left click on one of the newly divided sub-regions to assign it a new label. The other sub-region automatically inherits the old label.
Region Merging. This interaction assigns the same identity to components with similar content and texture. Users simply left click on all the regions to merge and confirm at the end to assign a consistent label. They can right click to cancel a selection before hitting the apply button. Note that regions to merge do not need to be adjacent.

We present the segmentation results before (Figure 6(a)) and after (Figure 6(d)) performing interactive region splitting and merging on the Bodhisattva image. As exemplified the zoom-in views, we separate the baldachin from the background (Figure 6(b)) and combine the hand and arm segments into one component (Figure 6(c)).

\subsection{Interactive Restoration}

To perform interactive restoration, the most essential step of the entire process, users need to select a target region, appoint a reference region from which the color and texture for inpainting are borrowed. User can choose to repaint the entire target region or just the selected damaged region extracted in the previous step.

If there is a large well-preserved area in the target region and the condition of the remaining parts is not too severe, the target region can serve as its own reference for restoration. If the target region is seriously damaged with only some small, discrete areas staying intact, it is better to employ another better-preserved region with similar content as the reference.

We extend the exemplar-based image inpainting algorithm proposed in (Criminisi et al., 2004) in our system. The whole inpainting process employs a best-filling-first strategy. That is, pixels with high-continuation and high-confidence neighbors will be painted first. We use a $R \times R$ patch window to fill the target region (indicated b $T$ ) with pixels from the reference region (indicated by $S$ )

As stressed in (Efros and Leung, 1999), the size of the $R \times R$ patch window can greatly influence the quality and efficiency of the inpainting algorithm. A large $R$ can shorten the processing time, but come to drab inpainting results. On the contrary, a small $R$ may increase the diversity and complexity of the textures but with low running speed. To find an appropriate patch size for our

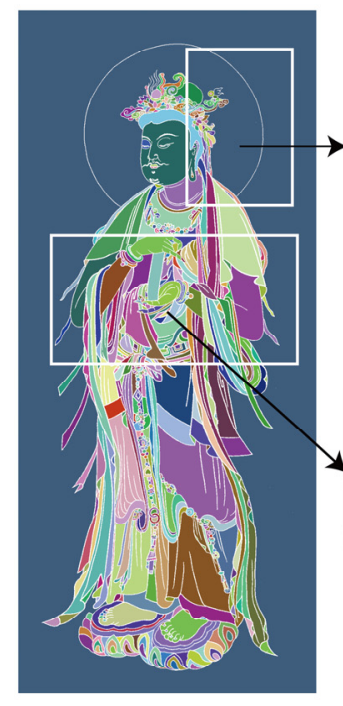

(a)

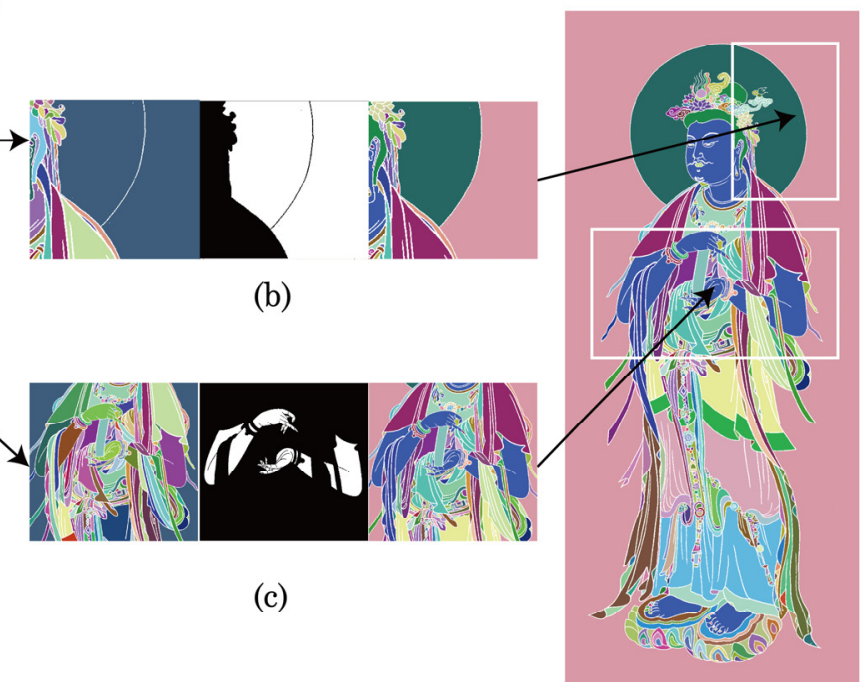

(d)

Figure 6. Segmentation result. (a) Original segmentation result; (b) Split baldachin region and background region; (c) Merge arms; (d) Optimized segmentation result. 
system, we evaluate the performance and efficiency of the inpainting algorithm with different $R$ on mural data from 1 to 25 . To balance the performance and efficiency and avoid obvious repetition of small patches appearing in the restoration result, we choose the default $R=6$. Our system also allows users to adjust the value of $R$ interactively, and provides a small sample result for each $R$ to facilitate this process.

Comparing our restoration result (Figure $4(\mathrm{e})$ ) to the original condition of the mural (Figure 4(c)), nearly all the obvious damages on the Bodhisattva figure are fixed. It shows that our system successfully reconstructs the integrated structure and harmonious color style of the mural.

\section{VALIDATION AND EVALUATION}

The approach we proposed leverages unique data, and the workflow is designed particularly for restoring Dunhuang murals Our system works in a way where its manipulations are similar to those in existing image editing software.

To understand how experts and amateurs may perceive and interact with our system, we conduct a preliminary user study of 15 users. Five experts from Dunhuang Research Academy in China and 10 amateurs majored in design or engineering are involved in our experiment. We assess the system from three aspects: 1) system performance, such as user satisfaction with the output; 2) interaction experience, such as learnability, ease of use, satisfaction, and task completion statues; and 3) interface design.

\subsection{Validation}

Currently, there is no standard measurement or parameterized method to validate the correctness of restored Dunhuang murals, especially for digital restoration. The validation of our system is done by two authoritative experts from Dunhuang Research Academy in China. One is a practical artist who has been studying and facsimileing Dunhuang murals for years, the other one is an associate research fellow from The Conservation Research Institute of Dunhuang Research Academy. They conclude three principles to validate restoration results according to the standard ones for physical restoration.

1. Originality. That means representative features should remain unchanged in the aspect of cultural heritage conservation;

2. Integrity. Spectators should feel the paintings are integrated when appreciating;

3. Recognizability. The restoration traces should be identified by experienced observation or instrumental detection.

They validate the digital results produced by our system according to the principles. They clarify that our overall effect is acceptable and reliable with the features of originality, integrity, and recognizability, partially beyond the limitation of physical restoration by repainting the missing part. From aspects of art aesthetic, they comment that the repainted effects correspond to practical painting knowledge and techniques, and the texture produced by flat-coating simulating and line-drawing overlapping are reasonable and naturally presented.

\subsection{Experiment Setup and Participants}

Our system is built with Python and Qt. It runs in the environment of Ubuntu 12.0.4 on a 14-inch Thinkpad-T430 laptop, within an Intel ${ }^{\circledR}$ Core ${ }^{\mathrm{TM}}$ i7-3520M @ 2.90GHz CPU, Intel ${ }^{\circledR}$ HD Graphics
4000 GPU, 8.00GB RAM for computing. We invited 15 users to attend our user experiment, and divided them into three groups by their backgrounds. We observed and video recorded their manipulations, and spontaneous comments throughout the course of the study.

Group 1: Specialists (P1-P5, 4 males, 1 female, age range 27-45 years old) who have been working at Dunhuang Research Academy for more than 2 years for physical restoration and conservation.

Group 2: Professional designers (P6-P10, 5 females, age range 23-25 years old) who are experts on image editing with five or more years of experiences.

Group 3: Engineers (P11-P15, 4 males, 1 female, age range 2431 years old) who have strong technical background but are less familiar with image processing and editing.

\subsection{Study Procedure}

The study aims to investigate both the user experiences and performance of each component of our system. Each participant is required to perform four tasks that correspond to the four functional operations of our system. The procedure can be described as follows:

Training: Before each session, we show the participants the system interface and detailed interactions related to the task on the computer. Then we ask them to repeat the operations and try a similar assignment.

Main task: After trained, the participants proceed to the main task. (Session 1) damaged area selection, i.e., picking out all the damaged regions in the given image; (Session 2) region splitting, i.e., separating the given area into two regions; (Session 3) region merging, i.e., combining two or three pre-determined areas; and (Session 4) interactive restoration, i.e., repairing a given region by specifying the reference and patch size. We recorded all the user interactions and took screenshots of the participants' results in each session.

Rating: Upon the completion of each task, participants rate the corresponding system component for its learnability, ease of use, and level of satisfaction on a five-point Likert Scale. We also interview them to collect their comments and feedback, especially in comparison with traditional restoration and other image-processing or graphics editing tools that they have used before.

\begin{tabular}{|c|c|c|c|c|}
\hline & & Learn & Interaction & Satisfaction \\
\hline \multirow{2}{*}{ Session1 } & mean & 4.87 & 3.73 & 3.73 \\
\cline { 2 - 5 } & $\mathrm{sd}$ & 0.34 & 0.93 & 0.93 \\
\hline \multirow{2}{*}{ Session2 } & mean & 4.00 & 3.60 & 3.87 \\
\cline { 2 - 5 } & $\mathrm{sd}$ & 1.10 & 1.02 & 1.09 \\
\hline \multirow{2}{*}{ Session3 } & mean & 4.60 & 4.20 & 4.00 \\
\cline { 2 - 5 } & $\mathrm{sd}$ & 0.61 & 0.98 & 0.97 \\
\hline \multirow{2}{*}{ Session4 } & mean & 4.33 & 3.73 & 3.93 \\
\cline { 2 - 5 } & $\mathrm{sd}$ & 0.70 & 0.68 & 0.93 \\
\hline \multirow{2}{*}{ Overall } & mean & 4.45 & 3.82 & 3.88 \\
\cline { 2 - 5 } & $\mathrm{sd}$ & 0.89 & 0.88 & 0.94 \\
\hline
\end{tabular}

Table 2. User rating for the system. Session 1-4 refer to damaged area selection, region splitting, region merging and interactive restoration. 


\subsection{Evaluation Results}

Overall, participants' reactions and feedback were positive, Table 2 shows the mean and standard deviation of rating scores of learnability, ease of use, satisfaction and the overall rating. All the participants considered the system very easy to learn (Avg. $=$ $4.45)$ and the interactions fairly intuitive (Avg. $=3.82$ ). They were quite satisfied with the results (Avg. $=3.88)$.

Participants found our digital mural restoration system quite unique within the use of line drawings. They suggested the feasibility of our system for two purposes: 1) assisting experts to perform professional physical restoration and protection, in a way that allows experts to preview the texture and color of crack virtually in advance before heading to physical tasks; 2) providing an engaging, explorative experience for amateurs without the restraint from rigorous academic restoration;

\section{DISCUSSION}

Limitation. Although the overall performance of our system is good according to the evaluation and validation results, it still has some limitations.

First of all, the use of line drawing for mural restoration is one of the novel parts of our approach, however, it may also become the bottleneck, as the quality and quantity of line drawing available affect the performance and scalability of our system. The second limitation is the tradeoff between efficiency and quality. Since it is difficult to achieve restoration results with good quality completely automatically, we have introduced many user interactions to guide the process. However, some of the interactions rely on subjective judgment, such as damage selection and reference appointment. It may take time for users to gain enough experiences to provide confident opinions rather quickly. Third, it may be challenging to invite multiple people to collaboratively restore a mural, as their choices of system configuration and manual intervention at each step may lead to very different results. How to combine individual results into a mural of consistent style and effect will be a tough task.

Potential Extension. Digital restoration of murals is a long-term task. The whole infrastructure can be extended to incorporate new features and applications. For example, we can take advantage of crowdsourcing to collect line drawing. It can make the whole process more cost-effective and attract more people to appreciate the beauty of Dunhuang murals. One of the practical applications of our work is to demonstrate the system in Art museums, inviting visitors to help restore murals. Furthermore, we can put the system online to involving the general public in preserving the treasure. Our system can also serve as an interactive platform that supports creativity and learning. Artists can create their own style of mural. Children can play scrawl games with mural fragments and learn about Dunhuang culture at the same time.

\section{CONCLUSION AND FUTURE WORK}

In this article, we present a novel line-drawing and patternenhanced infrastructure that supports digital restoration of damaged murals in Dunhuang Mogao Grottoes, western China. We describe each part of the proposed infrastructure with illustrations and examples, from data capture and pre-processing, database establishment, to interactive restoration via a system that integrates user interactions with algorithms. The results of a preliminary user study show that our system enable more efficient mural restoration with good quality for both experts trained in restoration and amateurs interested in cultural heritage conservation.

In the future, we will improve this work in several directions. First, we will apply our system to more mural images from different dynasties and with different damage conditions. Second, we will extend our system to support collaborative mural restoration. Third, we plan to improve the current interaction functions, especially for the region splitting task. In addition, we will add more image-editing related operations, such as dragging and layering, revocation, etc., to improve the interaction experiences.

\section{ACKNOWLEDGEMENTS}

This work is supported by the National Basic Research Program of China under Grant No. 2012CB725300 and the National Natural Science Foundation of China under Grant No. 61232013, 61373072. The authors thank La Tu, Wen Wei, Juan Du, Yanting Chen for their support.

\section{REFERENCES}

Achanta, R., Shaji, A., Smith, K., Lucchi, A., Fua, P., Susstrunk, S., 2012. SLIC superpixels compared to state-of-the-art superpixel methods. IEEE Transactions on Pattern Analysis and Machine Intelligence, 34(11), pp. 2274-2282.

Adams, F. and Bischof, L., 1994. Seeded region growing. IEEE Transactions on Pattern Analysis and Machine Intelligence, 16(6), pp. 641-647.

Amano, T., 2006. Correlation based image defect detection. 18th Int. Conf. Pattern Recognit, 1(1), pp. 18-21.

Baglioni, P., Dei, L., Pique, F., Sarti, G., Ferroni, E., 1997. New qutogenous lime-based grouts used in the conservation of limebased wall paintings. Stud. Conserv, 42(1), pp. 43-54.

Bertalmio, M., Vese, L., Sapiro, G. and Osher, S., 2003. Simultaneous structure and texture image inpainting. IEEE Trans. Image Process, 12(8), pp. 882-889.

Blazek, J., Zitová, B., Benes, M. and Hradilová, J., 2009. Fresco restoration digital image processing approach. 17th Eur. Signal Process. Conf. (EUSIPCO 2009), Eusipco, pp. 1210-1214.

Cahill, J., Silbergeld, J. and Tang, K., 2001. Chinese Art and Authenticity. Bull. Am. Acad. Arts Sci, 55(1), pp. 17-36.

Carretti, E., Salvadori, B., Baglioni, P. and Dei, L., 2005. Microemulsions and micellar solutions for cleaning fresco surfaces. Studies in Conservation, 1, pp. 50.

Chan, T. F. and Shen, J., 2002. Mathematical Models for local non-texture inpainting. SIAM, J. Appl. Math, 62(3), pp. 10191043.

Chang, R. C., Sie, Y. L., Chou, S. M. and Shih, T. K., 2005. Photo defect detection for image inpainting. Seventh IEEE Int. Symp. Multimed, pp. 403-407.

Chen, G., Fan, Z., Yu, Q., Su, B., Li, Y., Qiao, H., Tang, W., 2009. Primary test of desalination to treat the disrupted wall painting in Cang Jie Temple of Baishui, Shaanxi Province. Dunhuang Research, 6, pp. 6. 
Chen, G. and $\mathrm{Yu}, \mathrm{Z}$., 2008. Test for blister and soluble salt in powdering and the layer of plaster for the wall -painting in Cave 351 at Mogao Grottoes. Dunhuang Research, 6, pp. 39-45+119$120+116$.

Criminisi, A., Pérez, P. and Toyama, K., 2004. Region filling and object removal by exemplar-based image inpainting. IEEE Trans. Image Process. 13(9), pp. 1200-1212.

Drori, I., Cohen, D. and Yeshurun, H., 2003. Fragment-based image completion. ACM Trans. Graph, 22(3), pp. 303.

Efros, A. A. and Leung, T. K., 1999. Texture synthesis by nonparametric sampling. Comput. Vision. Proc. Seventh IEEE Int. Conf, 2(2), pp. 1033-1038.

Fan, J., Yau, D. K. Y., Elmagarmid, A. K., Aref, W. G., 2001. Automatic image segmentation by integrating color-edge extraction and seeded region growing. IEEE Transactions on Image Processing, 10(10), pp. 1454-1466.

Fan, Z. X., Chen, G. Q., Su, B. M., Li, R. F., Qiao, H., Tang, W., 2009. The testing study for repairing the disruption wall paintings in Cave 98 at Mogao Grottoes. Dunhuang Research, 6, pp. 4$7+120+125$.

Fan, Z. X., Rickerby, S., Shekede, L., Qiao, H., Tang, W., Yang, J. J., 2008. The technique measurement for repairing the mural paintings in Cave 85 at Mogao Grottoes. Dunhuang Research, 6, pp. 19-22.

Fraser, S. E., 2000. Formulas of creativity: artist's sketches and techniques of copying at Dunhuang. Artibus Asiae 59(3/4), pp. 189-224.

Giakoumis, I., Nikolaidis, N. and Pitas, I., 2006. Digital image processing techniques for the detection and removal of cracks in digitized paintings. Image Process. IEEE, 15(1), pp. 178-188.

Harrison, P., 2001. A non-hierarchical procedure for re-synthesis of complex textures. Proc. WSCG, 190-197.

Li, J. M., Zhang, H., Fan, Z. X., He, X., He, S. M., et al., 2013. Investigation of the renewed diseases on murals at Mogao Grottoes. Heritage Science, 1(1), pp. 31.

Li, Z. X., 2004. Sixty years on the conservation of the Dunhuang Grottoes. Dunhuang Research, 3, pp. 10-26+111.

Ma, Z. F. and Wang, W. F., 2014. On the materials and flaking of the wall paintings in Mogao Cave 44. Dunhuang Research, 5, pp. 108-118.

Mastio, A. D., Cappellini, V., Caldelli, R., Rosa, A. D. and Piva, A., 2007. Virtual restoration and protection of cultural heritage images. In Digital Signal Processing, 15th IEEE International Conference, pp. 471-474

Milanesi, C., Baldi, F., Vignani, R., Ciampolini, F., Faleri, C. and Cresti, M., 2006. Fungal deterioration of medieval wall fresco determined by analysing small fragments containing copper. Int. Biodeterior. Biodegrad, 57(1), pp. 7-13.

Padalkar, M. G. and Joshi, M. V., 2015. Auto-inpainting heritage scenes: a complete framework for detecting and infilling cracks in images and videos with quantitative assessment. Mach. Vis. Appl, 26(2-3), pp. 317-337.
Palmer, P., Dabis, H. and Kittler, J., 1996. A performance measure for boundary detection algorithms. Computer Vision and Image Understanding, 63(3), pp. 476-494.

Pappas, M. and Pitas, I., 2000. Digital color restoration of old paintings. IEEE Trans. Image Process, 9(2), pp. 291-294.

Paragios, N. and Deriche, R., 2000. Geodesic active contours and level sets for the detection and tracking of moving objects. IEEE Transactions on pattern analysis and machine intelligence, 22(3), pp. 266-280.

Ray, S. and Turi, R. H., 1999. Determination of number of clusters in k-means clustering and application in colour image segmentation. Proceedings of the 4 th international conference on advances in pattern recognition and digital techniques, pp. 137143.

Rosenfeld, A. and Pfaltz, J. L., 1966. Sequential operations in digital picture processing. Journal of the ACM,13(4), pp. 471494.

Russell, B., Sivic, J., Ponce, J., Dessales, H., 2011. Automatic alignment of paintings and photographs depicting a 3D scene. IEEE International Conference on Computer Vision Workshops, pp. 545-552.

Sayre, E. V., 1973. Investigation of Italian frescoes, their materials, deterioration and treatment. Application of science in examination of works of art-proceedings of the seminar: June 1519, 1970, conducted by the research laboratory, Museum of Fine Arts, Boston, Massachussetts, 1973, pp. 176-181.

Starck, J. L., Elad, M., Donoho, D. L., 2005. Image decomposition via the combination of sparse representations and a variational approach. IEEE Trans. Image Process, 14(10), pp. 1570-1582.

Szeliski, R., 2006. Image Alignment and Stitching: A Tutorial. Found. Trends ${ }^{\circledR}$ Comput. Graph. Vis, 2(1), pp. 1-104.

Tsai, A., Yezzi, A., Wells, W., Tempany, C., Tucker, D., Fan, A., Grimson, W. E., Willsky, A., 2003. A shape-based approach to the segmentation of medical imagery using level sets. IEEE Transactions on Medical Imaging, 22(2), pp. 137-154.

Turakhia, N., Shah, R. and Joshi, M., 2012. Automatic crack detection in heritage site images for image inpainting. Proc. Eighth Indian Conf. Comput. Vision, Graph. Image Process. ICVGIP '12, pp. 1-8.

Vincent, L. and Soille, P., 1991. Watershed in digital spaces:an efficient algorithm based on immersion simulation. IEEE Trans. Pattern Anal. Mach. Intell, 13(6), pp. 583-598.

Wang, W. F., Wang, T., Zhang, W. M., Zhang, G. B., Qiu, F., Zhan, H. T, 2000. Study on the design of a comprehensive sand drift control system at Mogao Grottoes, Dunhuang. Arid Land Geography, 20(4), pp. 409-414.

Whitfield, R., Whitfield, S. and Agnew, N., 2015. Cave Temples of Mogao at Dunhuang: Art History on the Silk Road (2nd. Ed.) . Getty Publications. 